\title{
Detection theory, micromatching, and the constant-ratio rule'
}

The conformance of detection theory and micromatching with the constant-ratio rule (CRR) was determined by calculations for sets of three stimuli and subsets of two stimuli. For an arbitrary selection of sets, the CRR would be expected to hold quite well if either detection theory or micromatching lay behind the responses. For specific stimulus configurations, however, detection theory and micromatching differ considerably from one another and from the CRR. These configurations are found particularly for the univariate distributions, and this corresponds to empirical findings that the CRR holds better for multidimensional than for unidimensional stimuli. It is shown how the differences between the three theories in the implied constancies of ratios can be used in diagnosis of the basis of empirical confusion matrices.

Consider the situation in which $\mathrm{S}$ must identify which of $\mathrm{N}$ possible known signals was randomly presented on a trial. This paper will discuss three theoretical approaches to this problem, and in particular will be concerned with a method distinguishing between them on the basis of confusion matrix data.

Detection. theory (decision theory applied to psychophysics) treats the problem by assuming that internal to $S$ there is a continuous probability distribution associated with each signal (stimulus) and that a random sample point from the distribution occurs on each trial when the signal is presented. Cutoff points, lines, or surfaces are set down by $\mathrm{S}$, dividing the total sample space into regions. Each region will receive the associated response; sample points occurring in one region will generally have derived from several signals (Swets, Tanner, \& Birdsall, 1961).

Lee (1963) suggested the possibility of maintaining the idea of distributions, but with a probabilistic mechanism to map sample points into responses. If the response probabilities conditional on sample position equal the sample-conditional probabilities that such samples derive from the various signals, the response mode was called micromatching. Micromatching can be justifjed for conditions of feedback, at least, as the result of a learning process (e.g., Lee, 1963, 1966; Lee \& Zentall, 1966). Micromatching or something like it might be conceived to arise also from variation in a cutoff boundary, since such variation would imply probabilistic responding to sample points at the same location.

Luce (1959) treated the problem without assuming distributions associated with each signal. According to an implication of his axiom 1 , when signal $s_{i}$ is presented, the ratio of the response probabilities conditional on that signal, $r_{j} / r_{k}$ (where $j$ or $k$ might equal i), has a value that is constant regardless of the presence or absence of any signals other than $i, j$, and $k$ that might be included in the signal set. Clarke (1957) independently proposed this same hypothesis and called it the constant-ratio-rule (CRR). A number of experimental studies have been devoted to determining the adequacy of the CRR. Clarke (1957) published results utilizing spoken syllables, words, and numbers, with highly favorable results. Luce (1959) took these results as the only findings bearing on the adequacy of his axiom 1 and considered the results to be highly favorable to his theory. Later studies have been somewhat less consistent. Hodge and Pollack (1962), e.g., differentiated cases in which the CRR was more, or less apt to hold. Multidimensional stimuli seemed to favor the CRR more than unidimensional ones.

The question arises as to the relationship between the CRR, detection theory, and micromatching in connection with the assumed constancies of response probability ratios. Is it the case, as for the shape of ROC curve (Lee, 1963; Luce, 1959), that the three are practically indistinguishable and that the constancy proposed for the CRR could be as well derived from detection theory and micromatching? Or is it the case that the constancy proposed by the CRR could not be consistent with detection theory, micromatching, or both? Does the degree of consistency implied by detection theory and micromatching differ? If so, perhaps the technique of calculating the degree of constancy might be diagnostic in differentiating theories such as detection theory and micromatching.

In order to answer these questions, a number of hypothetical stimulus configurations were examined to determine the degree of constancy that would be predicted from detection theory and micromatching.

\section{METHOD}

All the cases considered concern sets of three stimuli and subsets of two of these stimuli. It was assumed that whenever a stimulus is presented to an $S$ it gives rise internally to a random independent sample point from a normal (Gaussian) univariate or bivariate probability distribution. It was assumed in all cases that every stimulus in each set or subset was presented randomly and with equal probability. The standard deviations for all distributions in a set or subset were taken to be equal across stimuli and across dimensions. For bivariate stimuli, the covariance of the distributions was taken to be zero.

First, consider the univariate situations. The basic arrangement is shown in Fig. 1. Three normal distributions are located on a continuum. The distance between Distributions 1 and 2 equals the distance between 2 and 3. Different configurations were obtained by letting that distance vary. The vertical straight lines indicate the cutoff points for detection theory for three stimuli. These cutoffs divide the continuum into three regions, and the response given to a sample is assumed to be " 1 ," " 2 ," or " 3 ," depending on whether the sample is in the left, middle, or right region, respectively. These locations assume that the payoff matrix is such that $S$ essentially wishes to maximize the probability that his response is correct; e.g., $S$ might receive the same payoff for a correct response to any stimulus and a lesser payoff for an incorrect response to any stimulus. When the subset of two stimuli is employed, the comparable rationale implies that a single cutoff point should be set down midway between the means of the two distributions.

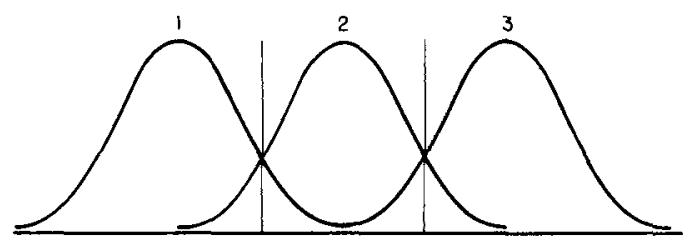

Univariate
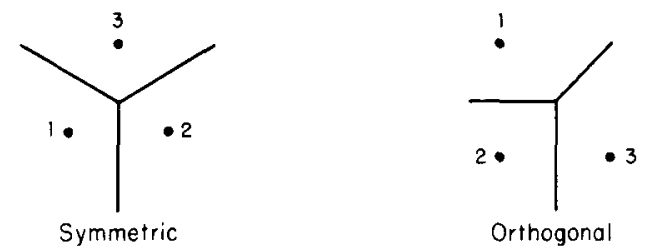

Fig. 1. The three arrangements of three normal distributions for which constancies were calculated. The univariate case illustrates the density functions, while the bivariate cases (symmetric and orthogonal) show only the relative locations of the means in two dimensions. The vertical lines in the univariate case indicate the cutoff points for the three-choice situation. The cutoff lines are shown for the three-choice symmetric and orthogonal cases. 
The two basic bivariate situations are also shown in Fig. 1. For these cases the probability densities are not shown, since the two dimensions of the paper represent the two dimensions of the sample space. For the "symmetric" case, the means are located at the vertices of an equilateral triangle. For the "orthogonal" case, the means are located at the vertices of an isosceles right triangle. Straight lines are shown that divide each two-dimensional space into response regions. Again it is assumed that payoffs are such that the task for $S$ is essentially to maximize the proportion of correct identifications. For both bivariate situations for detection theory, any sample falling in the region around Distribution 1 receives the response " 1 ," etc. When the subset of two stimuli is employed, a cutoff line is employed that is located midway between the distribution centers and that is perpendicular to a straight line joining the centers. (For our purposes, the two-stimuli situation can be treated as the univariate case derived by projecting each sample point onto the line joining the distribution centers.)

The conditional response probabilities for detection theory were determined by numerical integration on a computer of the densities for each distribution in each response region.

For micromatching for both the univariate and bivariate cases, the probability of response $i$ for the sample at any position is probabilistic, and is given by the conditional probability that a sample at that position derived from a given stimulus $s_{\mathrm{j}}$. That is, if .38 of the samples at a location derive from Distribution 2 , then the probability of response " 2 " for a stimulus at that position is .38. To get the stimulus-conditional response probabilities, one has to integrate over the entire space; there are no cutoff lines. Any response has some probability for any sample position, for the type of distributions specified.

For the three-stimulus bivariate case, the formula for the probability of response $j$, given $s_{i}$, is:

$$
r_{j}=\iint \frac{f_{j} f_{i}}{f_{1}+f_{2}+f_{3}} d x d y,
$$

where $f_{1}, f_{2}$, and $f_{3}$ are the bivariate probability densities. For the univariate cases and for cases of only two stimuli, the obvious simplifications of the formula are employed. The distance between means was varied to produce different cases.

The stimulus-conditional response probabilities were calculated for the three stimuli and for subsets of two stimuli. Conditional on the presentation of $s_{i}$ when the number of stimuli $N=3$, let the response probabilities be $r_{i}^{\prime \prime}, r_{j}^{\prime \prime}$, and $r_{k}^{\prime \prime}$, and let them be $r_{i}^{\prime}, r_{j}^{\prime}$, when $\mathrm{N}=2$. Then, by definition, the ratio

$$
\frac{r_{i}^{\prime} / r_{j}^{\prime}}{r_{i}^{\prime \prime} / r_{j}^{\prime \prime}}=c_{i j}
$$

Call c the "constancy." According to the CRR, $c_{i j}=1.0$; however, as we shall see, there is no necessity that $c_{i j}=1.0$ for detection theory or micromatching. Let

$$
\frac{r_{j}^{\prime} / r_{i}^{\prime}}{r_{j}^{\prime \prime} / r_{i}^{\prime \prime}}=c_{j i}
$$

It follows readily that

$$
c_{j i}=\frac{1}{c_{i j}}
$$

Obviously, then, if either of $c_{i j}$ or $c_{i j}$ is equal to 1.0 , both must be, and if either is greater than 1.0 , the alternative must be less than 1.0. $c_{i j}$ and $c_{i j}$ are redundant quantities, and which one to use is an arbitrary matter, but it is convenient to standardize on the one giving $c \leqslant 1.0$. For one thing, it is then possible to say "the constancy is greater" for one situation rather than another when the $c$ value for the first situation is greater than the $c$ value for the second. Henceforth, then, we drop the subscripts and assume that the response probability ratios are taken so that $\mathrm{c} \leqslant 1.0$.

The constancies were calculated for the cases in Fig. 1 for a variety of distances between means.

\section{RESULTS}

The calculated constancies are given in Table $1, \mathrm{~d}^{f}$ is the distance between distributions in terms of the common standard

deviation. The tabulated value of $\mathrm{d}^{\prime}$ is always the distance between the means for the subset of two stimuli; however, for $\mathrm{d}^{\prime}$ changes, all three stimuli were adjusted so that the relative positions of the three stimuli remained as shown in Fig. 1 ; i.e., in the univariate situation, as $d^{\prime}$ changed $s_{2}$ remained centered between $s_{1}$ and $s_{3}$. The constancies for $d^{\prime}=0.0$ are not calculations; the values are rational, and in the case of detection theory are limits as $\mathrm{d}^{\prime}$ approaches 0.0 .

All entries refer to the ratio of two stimulus-conditional response probabilities, given a subset of two signals, divided by the ratio of the same stimulus-conditional response probabilities, given the set of three signals. The signal on which the response probabilities are conditional is the same for both sets, and therefore, must be present in both the two- and three-signal sets. The two response probabilities and the sense of the ratio between them is indicated by, e.g., $r_{1} / r_{1}$. The signal on which the response probabilities are conditional is given following the colon. All possible ways of calculating constancies for the univariate situation are listed, except when the constancies would be inversions of those listed or would be equal by symmetry. Because of symmetry, only one constancy need be calculated in the "symmetric" case for each $d$ ". For the "orthogonal" case, only the condition $r_{1} / r_{3}$ : $s_{1}$ is left out, but this case would be similar to the "symmetric" case, with some deviation toward the constancies shown in the univariate case (2).

Note that most of the constancies are close to 1.0 , and for many situations the CRR would hold to a good approximation even if the postulates of detection theory or of micromatching lay behind the responses. On the other hand, for some situations, the constancy for detection theory, micromatching, or both differs considerably from 1.0 , in which case real data with some reasonable precision to it must lead to rejection of at least one of the three formulations, and possibly of two.

Micromatching generally produces constancies closer to 1.0 than detection theory. In fact, for an arbitrary selection of configurations, one would expect, on the basis of the calculations provided here, that if micromatching lay behind a set of responses, one would get a good fit to the CRR.

Also note that constancy holds up better for both detection theory and micromatching, as a rule, for the bivariate cases, than for the univariate cases.

The sense in which the constancy was taken to make $c \leqslant 1.0$, i.e., $c_{i j}$ or $c_{i j}$, did not have to be changed for the different values of $\mathrm{d}^{\prime}$ employed for any of the six cases. Nothing said so far

Table 1

Constancies for Detection Theory (DT) and Micromatching $\left(M_{\mu}\right)$

(1)

$\begin{array}{cc}\mathbf{r}_{1} / \mathbf{r}_{2}: & \mathbf{s}_{1} \\ \text { DT } & \mathbf{M}_{\mu} \\ 0.00 & 1.00 \\ 0.44 & 0.92 \\ 0.78 & 0.86 \\ 0.94 & 0.91 \\ 0.99 & 0.96 \\ 1.00 & 0.98 \\ 1.00 & 0.99\end{array}$

Symmetric

(4)

$r_{i} / r_{j}: s_{i}$

$$
(i \neq j)
$$

d'

0.0

0.5

1.0

1.5

2.0

2.5

3.0

Univariate

(2)

$\begin{array}{cc}\mathrm{r}_{1} / \mathrm{r}_{3}: & \mathrm{s}_{1} \\ \mathrm{DT} & \mathrm{M}_{\mu} \\ 1.00 & 1.00 \\ 0.96 & 0.99 \\ 0.85 & 0.97 \\ 0.68 & 0.93 \\ 0.51 & 0.84 \\ 0.34 & 0.74 \\ 0.22 & 0.62\end{array}$

Bivariate 
implied such a condition. The mathematical problem suggest:; itself of proving for each case whether or not the sense of the constancy would be the same for any d'value. Although I have not dwelled on the issue, affirmative proof for detection theory for case (1) resides in the formula, $c_{12}=r_{2}^{\prime \prime} /\left(r_{2}^{\prime \prime}+r_{3}^{\prime \prime}\right)$, and for case (3) in $c_{32}=r_{2}^{\prime \prime} /\left(r_{2}^{\prime \prime}+r_{1}^{\prime \prime}\right)$.

\section{DISCUSSION}

It seems clear from the table that if one were selecting cases arbitrarily, the CRR could be expected to hold quite well whether detection theory or micromatching were in actuality behind the responses. If one wished for a critical test, however, one would not choose cases arbitrarily, but would choose cases where the prediction of the CRR, detection theory, and micromatching differ considerably. These would be univariate cases, since here is where the notable differences occur, as far as can be told from the results at hand.

The use of Table 1 to compare detection theory and micromatching is not as straightforward as one might think, since if one is uncertain concerning which of these modes is behind the data for a set of two stimuli, then the value of $d^{\prime}$, given that $p \%$ of the responses in a yes-no detection task were correct, is not known. One value of $d^{\prime}$ would be inferred if detection theory were assumed, and another value of $\mathrm{d}^{\prime}$ would be inferred if micromatching were assumed. A graph in Lee (1963) gives the inferred $\mathrm{d}^{\prime}$ values for any percentage of correct identifications for detection theory and micromatching. The differences for the inferred $\mathrm{d}^{\prime}$ values amount only to about 0.5 at the most and are close to 0.5 over a large range of percentages, from about $55 \%$ to 92\%. The inferred $\mathrm{d}^{\prime}$ values assuming micromatching are close to 0.5 larger throughout this range of percentage correct responses than the inferred $\mathrm{d}^{\prime}$ values assuming detection theory. Suppose, e.g., $60 \%$ of the responses were correct in the two-choice situation involving $s_{1}$ and $s_{2}$ of case (1). Then, assuming detection theory, the value of $\mathrm{d}^{*}$ would be about 0.5 . The constancy expected would be about .44. On the other hand, assuming micromatching, the value of $\mathrm{d}^{\prime}$ would be about 1.0 , and the constancy expected would be about .86. Given that $60 \%$ of the responses were correct for the set of two stimuli, rather different values for the constancy would be predicted assuming detection theory or micromatching.

The results in Table 1 correspond to, and may help explain a generalization noted by Hodge and Pollack (1962) and Hodge (1967): the CRR does better for the multidimensional stimuli than for the unidimensional stimuli. There is nothing in Luce's choice theory to indicate such a result, nor did Clarke indicate any such expectation in his original presentation of CRR. Clarke later put forward doubts on the adequacy of the CRR for unidimensional stimuli, but these doubts were based on empirical work. The present results perhaps give some notion as to why the CRR fares better with multidimensional stimuli: with either detection theory or micromatching (or presumably something in between them), constancy is greater for the univariate cases than for the bivariate cases. Of course, the number of cases calculated for the univariate and bivariate conditions was not very large and did not allow, e.g., for different standard deviations, but the trend seems unmistakable on the basis of the cases seen.

It must be recognized that neither detection theory nor micromatching, as presently formulated, can constitute a basis for an adequate psychophysical theory of confusion matrices for unidimensional stimuli: One of the more noteworthy findings of recent psychophysical research is that the amount of information transmitted in unidimensional absolute judgment tasks reaches a maximum with a relatively small number of stimuli-about five in the case of loudness or pitch (Garner, 1962, Chap. 3). The unidimensional stimulus identification task is a kind of absolute judgment task, and therefore the amount of information that can be transmitted must have severe limitations. Neitlier detection theory nor micromalching allows for such a limitation; either would imply that continuously increasing values of information transmission could be obtained by successive increases in the number of stimuli and their range. It may be, however, that a comparison of detection theory and micromatching makes sense providing that the number of stimuli utilized never exceeds a maximum, which might be the maximum number of stimuli that can be perfectly identified in absolute judgment experiments for the continuum of interest.

It is not held that detection theory could not yield any other constancies than the ones given. For example, by allowing "noise," or "error," to enter into the location of the cutoff points and lines, the predictions could be made more like those for micromatching. Likewisc, detection theory predictions have been modified by the inclusion of other factors, such as memory (Tanner, 1961). Such possible modifications lead to numerous and complicated possibilities, which have not been considered in the present study. Likewise, various modifications of the idea of a "stochastic boundary," such as seen in the idea of micromatching, might be investigated, and these would yield different constancies. Once such variations are specified, however, the constancies implied can be calculated, and these could then be used in diagnosis.

One need not expect that all.Ss would have the same pattern of constancies. If the results with externally distributed stimuli are any indication (Lee, 1963; Lee \& Janke, 1964, 1965), some Ss might use rather sharp cutoff boundaries, and others might not.

In view of the implied effects of the individual stimulus configurations on constancy, I would suggest that investigators of the CRR give more detail on individual response probabilities for specific configurations, rather than simply giving gross plots and gross statistics. If this level of detail is not possible for a journal article, at least the availability of the data could be advertized.

\section{REFERENCES}

ClARKE, F. R. Constant-ratio rule for confusion matrices in speech communication. J. Acoust. Soc. Amer., 1957, 29, 715-720.

GARNER, W. R. Uncertainty and structure as psychological concepts. New York: Wiley, 1962.

HODGE, M. H. Some further tests of the constant-ratio-rule. Percept. \& Psychophys., 1967, 2, 429437.

HODGE, M. H., \& POLLACK, I. Confusion matrix analysis of single and multidimensional auditory displays.J. exp. Psychol., 1962, 63, 129-142.

LEE, W. Choosing among confusably distributed stimuli with specified likelihood ratios. Percept. mot. Skills, 1963, 16, 445-467.

LEE, $W$. Conditioning parameter model for reinforcement generalization in probabilistic discrimination learning. J. math. Psychol, 1966, 3, 184-196.

LEE, W., \& JANKE, M. Categorizing externally distributed stimulus samples for three continua. J. exp. Psychol., 1964, 68, 376-382.

LEE, W., \& JANKE, M. Categorizing extemally distributed stimulus samples for unequal molar probabilities. Psychol. Rep., 1965, 17, 79-90.

LEE, W., \& ZENTALL, T. R. Factorial effects in the categorization of externally distributed stimulus samples. Percept. \& Psychophys., 1966, 1, 120-124.

LUCE, R. D. Individual choice behavior. New York: Wiley, 1959.

SWETS, J. A., TANNER, W. P., JR., \& BIRDSALL, T. G. Decision processes in perception. Psychol. Rev., 1961, 68, 301-340.

TANNER, W. P., JR. Physiological implications of psychophysical data. Ann. N.Y. Acad. Sci., 1961, 89, 752-765.

\section{NOTES}

1. This research was supported by NIH Grant 11128

2. Now at Bell Telephone Laboratories, Holmdel, New Jersey.

(Accepted for publication June 10, 1968.) 